\title{
TRANSFORMACIÓN SOCIOECOLÓGICA EN EL AGROECOSISTEMA CAFÉ AFECTADO POR ROYA EN CHIAPAS, MÉXICO
}

\author{
SOCIO-ECOLOGICAL TRANSFORMATION IN THE COFFEE \\ AGROECOSYSTEM AFFECTED BY RUST IN CHIAPAS, MÉXICO
}

\author{
Esteban Escamilla-Prado*, Juan Á. Tinoco-Rueda', Hugo Alberto Pérez-Villatoro' ${ }^{1}$, Ángel \\ de Jesús Aguilar-Calvo', Rufo Sánchez-Hernández ${ }^{2}$ y Diana Ayala-Montejo ${ }^{3}$
}

\author{
'Universidad Autónoma Chapingo (UACh), Centro Regional Universitario Oriente, Huatusco, Veracruz, México. ²Universidad Juárez Autónoma de \\ Tabasco, División Académica de Ciencias Agropecuarias, Ranchería La Huasteca, Villahermosa, Tabasco, México. ${ }^{3}$ UACh, Chapingo, Estado de \\ México, México. \\ *Autor de correspondencia (espreschoca@yahoo.com.mx)
}

\section{RESUMEN}

El agroecosistema de café (Coffea arabica) proporciona servicios ambientales, identidad cultural y sustento económico a caficultores en el estado de Chiapas, principal estado productor de café en México. Con la llegada de la roya (Hemileia vastatrix) en el año 2012, estos sistemas agroforestales se modificaron. El objetivo de la presente investigación fue analizar la respuesta del sistema a dichos cambios, relacionados con el proceso de transformación socioecológica a través de las respuestas estratégicas adoptadas por los productores para enfrentar los problemas ocasionados por la roya. Se realizó una caracterización agroecológica, agronómica y socioeconómica del cafetal. Los resultados revelan que los productores corresponden a una población de edad avanzada, de bajo nivel escolar, minifundistas, que pertenecen a diferentes grupos étnicos. Los agroecosistemas se desarrollan mayormente en altitudes intermedias, entre 1000 y 1300 msnm, que se manejan bajo esquemas agroecológicos y el 62.3 $\%$ son manejados como sistemas de policultivos tradicionales que mantienen un dosel de sombra y conservan la biodiversidad. La variedad Bourbon domina sobre las demás con $19 \%$. Se registró un aumento de la productividad en 10 de las 12 regiones analizadas durante el periodo 2015-2017. Se concluye que la estrategia de manejar diversas variedades de café bajo los sistemas de policultivo tradicional y sistemas especializados en el agroecosistema de café ha permitido su resiliencia a problemas fitosanitarios como la roya, además de contribuir a la recuperación de la productividad, y que se conserve como un patrimonio biocultural que ofrece servicios ambientales y sostiene económicamente a grupos de caficultores que custodian estos sistemas de alto valor agroecológico.

Palabras clave: Coffea arabica, agroecología, caficultura, fitosanidad, resiliencia, sistemas agroforestales.

\section{SUMMARY}

The coffee (Coffea arabica) agroecosystem provides environmental services, cultural identity and economic livelihoods to coffee growers in the state of Chiapas, the main coffee-producing state in Mexico. With the arrival of rust (Hemileia vastatrix) in 2012, these agroforestry systems were modified. The objective of this research was to analyze the response of the system to these changes related to the process of socio-ecological transformation, through the strategic responses adopted by producers to face the problems caused by rust. An agroecological, agronomic and socioeconomic characterization of the coffee plantation was carried out. Results revealed that the producers correspond to an elderly population, with a low educational level, smallholders who belong to different ethnic groups. Agroecosystems develop mostly at intermediate altitudes, between 1000 and 1300 masl, which are managed under agroecological schemes and $62.3 \%$ are managed as traditional polyculture systems that maintain a shade canopy and conserve biodiversity. The Bourbon variety dominates over the others with $19 \%$. An increase in productivity was recorded in 10 of the 12 regions analyzed during the 20152017 period. It is concluded that the strategy of managing diverse coffee varieties under traditional polyculture systems and specialized systems in the coffee agroecosystem has allowed their resilience to phytosanitary problems such as rust, in addition to contributing to the recovery of productivity, and that it is preserved as a biocultural heritage that offers environmental services and financially supports groups of coffee growers who are custodians of these systems of high agroecological value.

Index words: Coffea arabica, agroecology, agroforestry systems, coffee growing, plant health, resilience

\section{INTRODUCCIÓN}

El estado de Chiapas es el principal productor de café en México, con una superficie de 253,764 ha, equivalente al $36.8 \%$ de la superficie nacional de este cultivo. Tiene un padrón de 180,856 productores, mayormente campesinos indígenas minifundistas, que aportaron el $40.4 \%$ de la producción nacional (INCAFECH, 2020). Chiapas es escenario de organizaciones cafetaleras campesinas que aportan a la cadena de valor y permiten a los caficultores obtener mayores ingresos a través de la comercialización de cafés diferenciados (Naylor, 2017); sin embargo, en 2012 la llegada de la roya (Hemileia vastatrix) al sureste mexicano provocó una importante caída en la producción de hasta 65 \% (Libert-Amico y Paz-Pellat, 2018), dicha problemática fue parte de una situación generalizada que ocurrió en Centroamérica en los años 2012-2015, donde la enfermedad ocasionó entre 10 y $55 \%$ de pérdidas en la producción en café arábica (Cerda et al., 2017). La combinación de los efectos de la enfermedad, con una 
baja en los precios internacionales del café, causó daños a la economía campesina de pequeños caficultores.

A pesar de las afectaciones de la roya y las condiciones desfavorables del mercado, los pequeños productores continúan cultivando café, no sólo porque constituye la principal fuente de ingresos, sino porque es parte de la identidad cultural de los pueblos. Según Merlin-Uribe et al. (2018), en la Sierra Madre de Chiapas, el sexo, edad y nivel tecnológico son elementos que definen los sistemas de producción, es así que las mujeres hacen uso de mano de obra permanente de origen externo, y el manejo es de baja intensidad productiva, conservan variedades tradicionales, con uso limitado de insumos y prácticas culturales, aunque se observa que, durante la cosecha, la demanda de mano obra aumenta; así mismo, la baja intensidad agroindustrial y alto uso de mano de obra temporal son características de la caficultura campesina y responde a estrategias de supervivencia.

La afectación de la roya causó cambios drásticos en el agroecosistema cafetalero en Chiapas, en particular, en las características del componente arbóreo del sistema agroforestal, así como en la sustitución de variedades tradicionales, ya que éstas son menos resistentes a la roya, menos productivas y más exigentes a la sombra (Henderson, 2019). La magnitud de dichos cambios no ha sido ampliamente abordada, por lo que la presente investigación tuvo como objetivo realizar el análisis de la transformación socioeconómica, técnica y agroecológica de los agroecosistemas de café en Chiapas, con el fin de evidenciar las diversas estrategias de los pequeños productores para adaptarse a la problemática ocasionada por la roya durante los ciclos productivos 2015-2016 y 2016-2017.

\section{MATERIALES Y MÉTODOS}

\section{Área de estudio y método de muestreo}

La investigación se realizó entre noviembre de 2016 y abril de 2017 en las regiones cafetaleras de Chiapas. Los productores por cada región se seleccionaron a través de un muestreo estratificado en cada estrato altitudinal, a partir de la cantidad de productores reportado por el Instituto del Café de Chiapas (INCAFECH) y la superficie cultivada con café. Con dicha información se definió un factor de importancia para asignar el tamaño de muestra. Se determinaron tres franjas altitudinales: 500-1000 msnm (zona baja), 1001-1500 msnm (zona media) y 1501 1900 msnm (zona alta). Según el INCAFECH (2020), 12 regiones productoras cuentan con los tres tipos de franjas altitudinales: Ocosingo, Ángel Albino Corzo, San Cristóbal, Motozintla, Comitán, Yajalón, Ocozocoautla, Bochil,
Mapastepec, Tapachula, Copainalá y Pichucalco (Cuadro 1), las cuales abarcan 29 municipios y 67 localidades. En estas regiones se estudió una muestra de 138 productores.

\section{Acopio de información}

A partir de información del padrón del INCAFECH (2020), se analizaron mediante estadística descriptiva las variables de producción: rendimiento de cereza ha-1 ${ }^{-1}$, cantidad de plantas ha-1 y área de cada productor, durante los ciclos productivos 2015-2017. Se recabó información a través de una encuesta sobre aspectos socioeconómicos como edad, escolaridad, población indígena, superficie cultivada, tenencia de la tierra, sistema de producción (convencional u orgánica) y aspectos agroecológicos y agronómicos como altitud, sistemas de cultivo, edad de los cafetales, diagnóstico de la estructura productiva (DEP), variedades cultivadas, prácticas de manejo (arvenses, tejido, nutrición y sombra), conservación de suelos, presencia de plagas y enfermedades, así como registros de productividad en los ciclos productivos 2015-2016 y 2016-2017.

\section{Caracterización agroecológica}

La caracterización agroecológica del cafetal se realizó mediante recorridos del predio en compañía del productor, con quien se ubicaron sitios representativos basados en el relieve, distribución de la sombra, especies y cantidad de árboles de sombra, variedades cultivadas y manejo del huerto. Con una cuerda, se delimitaron sitios de muestreo de $25 \times 25 \mathrm{~m}\left(625 \mathrm{~m}^{2}\right)$ y se georeferenciaron las coordenadas geográficas latitud, longitud y altitud con un equipo GPS (Marca Garmin, Garmin Corporation, Olathe, Kansas, EUA ), de acuerdo con la metodología propuesta por Escamilla et al. (2005). Se realizaron 100 mediciones por sitio de muestreo para determinar el porcentaje de cobertura arbórea y sombra con un densitómetro. En cada sitio se realizaron inventarios de las especies arbóreas que se usan como sombra y se caracterizó el sistema de cultivo; la caracterización consistió en identificar las especies de árboles de sombra, la densidad y distanciamiento, para determinar al tipo de sistema que corresponde, según la clasificación propuesta por Moguel y Toledo (1999), la cual se basa en la densidad de sombra.

Dentro del área de muestreo se eligieron al azar 25 plantas de café, se midió la distancia entre plantas y entre surcos para calcular la densidad de la plantación. Se realizaron diagnósticos visuales del estado fitosanitario de las plantas, deficiencias nutrimentales y las variedades.

\section{Análisis de la información}

Los datos se analizaron mediante el software AgriTask 


\begin{tabular}{lcc}
$\begin{array}{l}\text { Cuadro 1. Productores } \\
\text { regiones de Chiapas, México. }\end{array}$ & Número de & estudiados en las \\
\hline $\begin{array}{l}\text { Región } \\
\text { cafetalera }\end{array}$ & $\begin{array}{c}\text { puctuctores/cafetales } \\
\text { produje }\end{array}$ & \\
\hline Ocosingo & 38 & 28.3 \\
$\begin{array}{l}\text { Ángel Albino } \\
\text { Corzo }\end{array}$ & 15 & 10.9 \\
San Cristóbal & 15 & 10.9 \\
Motozintla & 15 & 10.1 \\
Comitán & 13 & 9.4 \\
Yajalón & 10 & 7.2 \\
Ocozocoautla & 9 & 6.5 \\
Bochil & 7 & 5.1 \\
Mapastepec & 5 & 3.6 \\
Tapachula & 5 & 3.6 \\
Copainalá & 3 & 2.2 \\
Pichucalco & 3 & 2.2 \\
Total & 138 & 100 \\
\hline
\end{tabular}

y el Programa Excel; para la comparación de sistemas se utilizó estadística descriptiva.

\section{RESULTADOS Y DISCUSIÓN}

\section{Perfil socioeconómico de los productores}

La edad de los productores oscila entre 19 y 89 años, con promedio de 50 años, y sólo el $7.2 \%$ de la población es menor de 30 años. Los productores de mayor edad están en Ocozocoautla y Tapachula, con edades promedio de 65 y 63 años respectivamente. La población más joven es la de Bochil, con 38 años de edad en promedio. Se observa que las personas de mayor edad son las que manejan los cafetales, así como la inexistencia de un relevo generacional entre los productores, debido a la desvinculación de los jóvenes con la caficultura, la migración, o por dedicarse a actividades no agrícolas. Al respecto Escamilla et al. (2005) reportaron que, en particular, los agroecosistemas de café orgánico en México son manejados por caficultores cuya edad promedio es de 49 años.

El nivel de escolaridad entre la población de caficultores es bajo, $15.2 \%$ no cursaron algún grado escolar, $40.5 \%$ no terminaron la primaria y sólo $5 \%$ concluyó estudios profesionales. Esos bajos niveles de escolaridad y el analfabetismo son fenómenos que provocan desigualdad económica y social, referida a la producción y distribución de bienes y servicios básicos establecidos por CONAPO, porque afectan el desarrollo pleno de las personas y su participación en la sociedad y el entorno familiar; además, obstaculizan el goce de otros derechos como el acceso a servicios básicos de luz y conectividad a internet, acceso a crédito, entre otras, particularmente en la población de mayor edad (Vargas, 2007).

Las superficies de las parcelas oscilan entre 1.2 y 6 ha, con promedios de 3.0 ha. Las parcelas con menor promedio de superficie son las de Bochil (1.2 ha), San Cristóbal (1.5 ha) y Yajalón (1.5 ha); las de mayor superficie son las de Mapastepec (6 ha) y Motozintla (4.7 ha). La tenencia de la tierra de estas parcelas es ejidal, pequeña propiedad y comunal con $72.4,21$ y $6.5 \%$ respectivamente.

La tipología de la producción se caracterizó por la presencia de sistemas de producción convencional y orgánica en 51.5 y $48.5 \%$, respectivamente. El mayor porcentaje de unidades de producción convencional se concentra en Copainalá, Ocozocoautla, Mapastepec y San Cristóbal de las Casas (Figura 1). La producción orgánica predomina en Yajalón, Ángel Albino Corzo y Bochil.

\section{Presencia de plagas y enfermedades}

La principal enfermedad identificada fue la roya del cafeto (Hemileia vastatrix) con $43.1 \%$, seguida de ojo de gallo (Mycena citricolor) con $15 \%$, mancha de hierro (Cercospora coffeicola) con $10.6 \%$ y con menos de $1 \%$ la antracnosis (Colletotrichum gloeosporioides), mal de hilachas (Corticium koleroga) y requemo (Phoma costarricenses) (Figura 2).

La presencia de daños por roya (Hemileia vastatrix) fue mayor en las regiones de Mapastepec (81.6\%), San Cristóbal de las Casas (78.1\%), Copainalá (74.7\%), Bochil (69.1\%) y Tapachula (68.8\%). Otras enfermedades con presencia importante en las regiones estudiadas son ojo de gallo y mancha de hierro, ambas causadas por hongos. La primera se identificó en mayor porcentaje en Bochil (50.3\%), Motozintla (26.3\%) y Ocosingo (21.7\%). En cuanto a la mancha de hierro, destacan las regiones de Mapastepec (28\%), Comitán (27.4\%), Ángel Albino Corzo $(21.3 \%)$ y Motozintla (18\%).

Las plagas presentes fueron minador de la hoja (Leucoptera coffeella) con $21.4 \%$, saltamontes (Orthoptera: Acrididae) con $7.6 \%$ y broca (Hypothenemus hampei) con $2 \%$ de incidencia (Figura 3). En menor proporción se encontraron daños de araña roja (Oligonichus sp.), gallina ciega (Phyllophaga sp.), hormiga arriera (Atta sp.) y escamas (Hemiptera: Coccidae), y dentro de los roedores la tuza (Orthogeomys sp.). El 


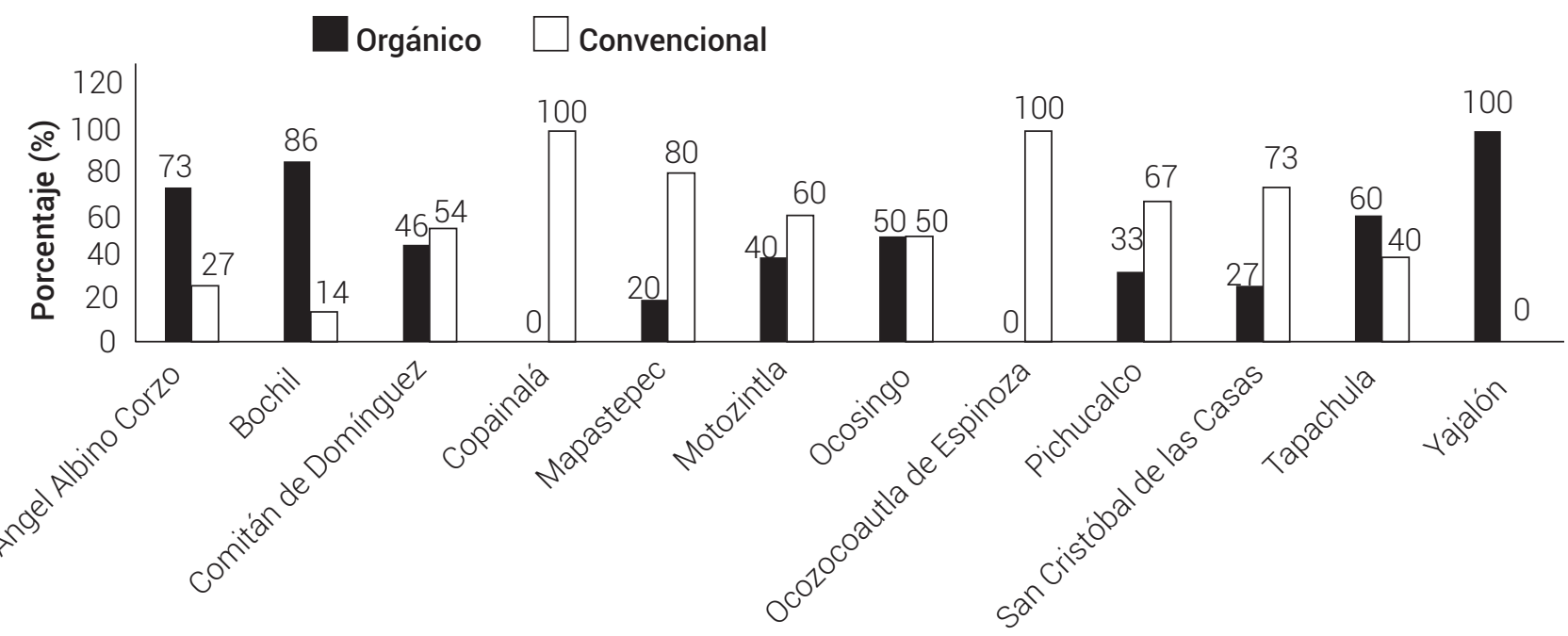

Figura 1. Distribución de tipos de sistemas de producción de café en las regiones de Chipas, México.

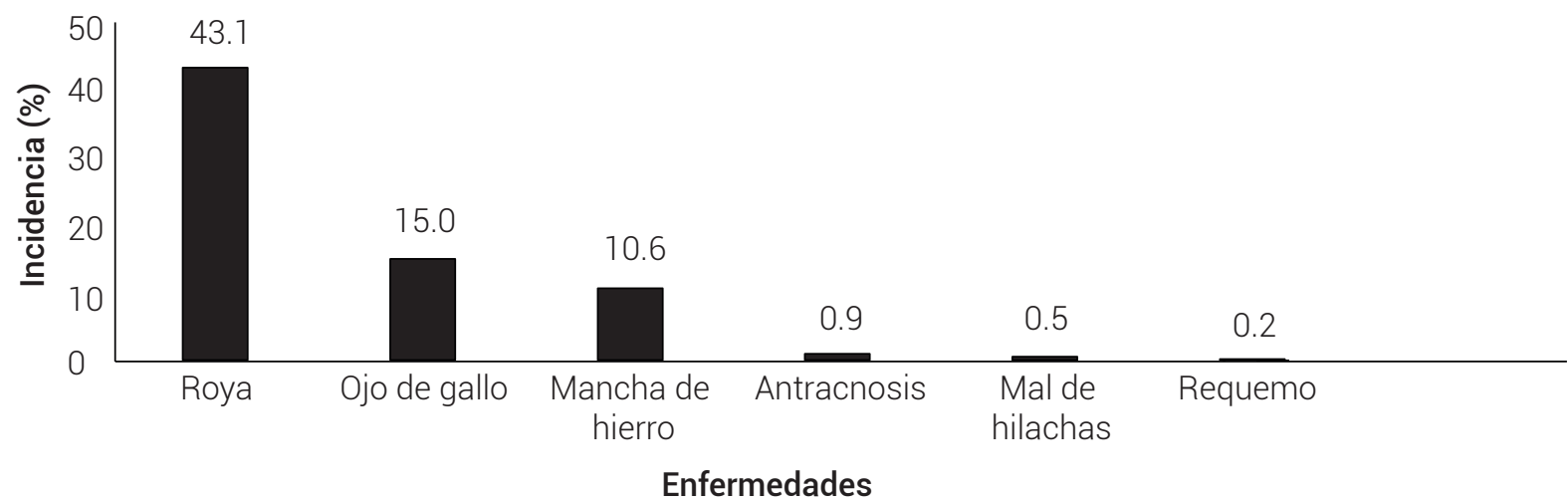

Figura 2. Enfermedades encontradas en los cafetales estudiados en Chiapas, México.

minador tiene mayor presencia en Ocozocoautla (51.1\%), Copainalá (36\%), Tapachula (35.2\%), San Cristóbal (33.9 $\%)$ y Mapastepec (31.2\%). Los saltamontes destacan en Bochil (25.7\%) y la broca del grano en Motozintla (5.2\%) y Mapastepec (3.5\%).

Técnicas adoptadas y manejadas en los agroecosistemas de café afectados por roya

\section{Manejo de la nutrición}

Se encontró que 53.6 \% de los caficultores suministran nutrientes a través de la fertilización y el abonado con composta, lombricomposta, lixiviados y sulfomagro; se encontró que 48.5 \% tienen certificación orgánica. En las regiones de Yajalón, todos los productores reportaron aplicación de nutrientes, Motozintla ocupa el segundo lugar con 93.8 \%, lo que concuerda con Escamilla et al. (2015), quienes reportaron que $75 \%$ de los productores orgánicos de Chiapas aplican abono cada año, mientras que Bochil (30 \%) y Tapachula (20\%) fueron las regiones con menos aplicación, en estas parcelas se logró identificar de manera visual la deficiencia de algunos nutrientes, destacando las deficiencias de nitrógeno, potasio y magnesio.

\section{Conservación de suelos}

En este estudio se encontró que las regiones de Tapachula y Mapastepec destacan por la utilización de barreras vivas, práctica que se realiza en el $60 \%$ del total de parcelas muestreadas. La conservación de suelos es un tema de mucha trascendencia para el cultivo del café; por 
ello, es necesario que este agroecosistema fortalezca su sostenibilidad en las laderas con un adecuado sistema de técnicas y prácticas de conservación de suelos. En el caso de Chiapas, la sombra y el manejo del cultivo contribuyen a proteger los suelos. Todos los caficultores orgánicos reportaron que realizan prácticas de conservación de suelos, en especial, el establecimiento de barreras vivas y los deshierbes o chapeos altos, entre 5 a $10 \mathrm{~cm}$ (Escamilla et al., 2005); por el contrario, en las regiones de Copainalá y Ocozocoautla no se realizan actividades de conservación de suelos.

\section{Sistemas de cultivo}

Los resultados del estudio indican que los sistemas de cultivo predominantes en Chiapas son sistema policultivo tradicional (SPT) y sistema especializado (SE) (Figura 4); en el primer caso, el sistema integra árboles de vegetación nativa y secundaria con árboles frutales nativos e introducidos; en el segundo caso, la sombra predominante se caracteriza por un dosel del género Inga, conocido localmente con los nombres de chalum, tzelel, paterno, entre otros. En ambas modalidades, el nivel de sombra en el cafetal alcanza hasta $97.8 \%$. Según Escamilla et al. (2005), los SPT y SE son los más utilizados en la producción de café orgánico en México. El $62.3 \%$ de los cafetales estudiados presenta la modalidad de sistema policultivo tradicional (SPT), siendo las regiones de Bochil, Ocozocoautla y Yajalón las que destacan por tener dicho sistema en $100 \%$ de los cafetales. El 30.4\% corresponde al sistema especializado (SE), que se caracteriza por poseer un dosel de especies de sombra del género Inga. El tercer lugar lo ocupa el sistema en policultivo comercial (SPC), con un total de $3.3 \%$, donde se intercala café con otros cultivos destinados al mercado, que contribuyen a complementar los ingresos de los productores. El sistema a pleno sol (SPS) y el sistema de montaña o rusticano (SR), sólo representan 2.1 y $1.8 \%$, respectivamente, del total de parcelas estudiadas. Estas diferencias coinciden con los resultados de Escamilla et al. (2005), quienes señalaron que los policultivos (SPT y SPC) son los sistemas de café orgánico que predominan en México, los cuales son manejados por los caficultores minifundistas, que se caracterizan por aplicar diversas estrategias de manejo agronómico de acuerdo con su experiencia y conocimientos, para el aprovechamiento integrado de todos los componentes del sistema agroforestal.

El $52.3 \%$ de los productores tienen un SPT que se encontró en casi todas las regiones, con excepción de la región de Pichucalco, siendo las regiones de Bochil, Ocozocoautla y Yajalón las que presentaron el $100 \%$ de SPT (Figura 5). Estos resultados coinciden con lo reportado por Escamilla y Díaz (2016), quienes mencionan que el SPT es dominante en las regiones Norte, Selva y Centro (Ocozocoautla y Cintalapa) y, frecuentemente, en algunos municipios cafetaleros del Soconusco, como Tuxtla Chico, Huehuetán, Huixtla, Mapastepec y Pijijiapan, y otras regiones del estado de Chiapas (Altos, Fronteriza y Frailesca), mientras que el SR se identificó en $1.8 \%$ de los cafetales visitados en la región de Ángel Albino Corzo con un total de 13.3 \% y también en San Cristóbal, Mapastepec y Motozintla. Escamilla y Díaz (2016) mencionaron que, en el siglo pasado, el SR fue importante en la Sierra Norte, en las regiones de Bochil y Mapastepec (en ésta llegó a representar $30 \%$ de los predios); sin embargo, este sistema mostraba tendencia a disminuir, tal y como se confirma en este estudio.

El segundo sistema con mayor presencia corresponde

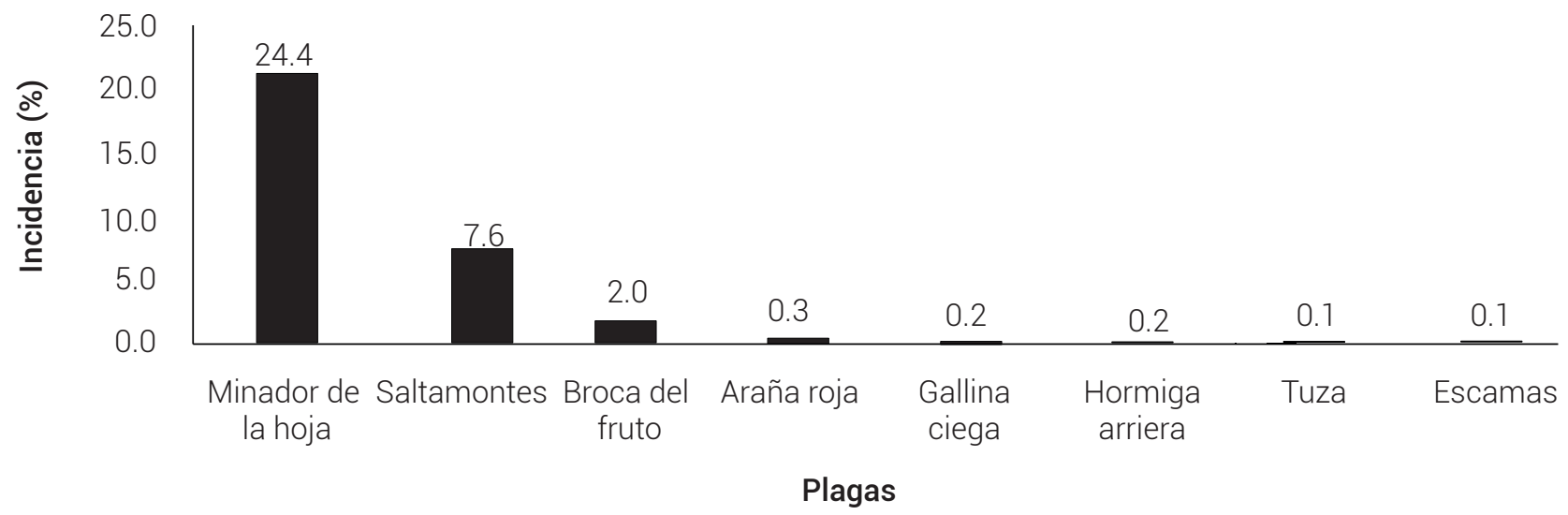

Figura 3. Plagas encontradas en los cafetales en Chiapas, México. 
al SE (30.4\%), distribuido principalmente en la región de Pichucalco, seguida de San Cristóbal (54.7 \%), Comitán (46.2 \%) y Ángel Albino Corzo (40\%). Este sistema fue impulsado por el Instituto Mexicano del Café (INMECAFÉ) a inicios de los años 1970s, con gran aceptación en la región del Soconusco, donde llegó a representar $80 \%$ de los cafetales, en especial en los municipios de Unión Juárez, Cacahoatán, Villa Comatitlán, Escuintla, Motozintla y Mapastepec; también fue muy importante en Ocosingo, Chilón, Altamirano y Sitala, teniendo menor presencia en las regiones Fronteriza, Centro, Selva y Norte (Escamilla y Díaz, 2016).

\section{Manejo del tejido}

El $97 \%$ de los productores entrevistados en Chiapas realizan poda. El método más frecuente es la poda selectiva (79.9\%), también se llevan a cabo actividades de agobio (61.4\%), recepa (56.9\%), deshije (55\%) y descope (20.1\%), mientras que los tipos de podas menos comunes son la poda pulmón (2.2\%) y el esqueletamiento (2.2\%). A nivel de sistemas de poda, $80.1 \%$ la realiza por plantas, $16.4 \%$ por surco y $5.4 \%$ por lote. Los resultados del estudio son parecidos a los reportados en la producción orgánica en México, donde $82 \%$ de los productores realizan poda, y de éstos, $46 \%$ efectúa recepa o poda profunda; en tanto que, $40.5 \%$ Ileva a cabo el sistema de poda tradicional, individual y selectiva, denominada "veracruzana" (Escamilla et al., 2005), la cual consiste en eliminar parcial o totalmente, y en diferentes grados de intensidad, los tallos (ejes) y ramas agotados o poco productivos.

\section{Densidad de plantación}

Las regiones de Ángel Albino Corzo y Motozintla tienen los cafetales con más altas densidades; en contraste, las regiones de Bochil y Pichucalco presentaron las menores densidades, de acuerdo con la estimación realizada durante los recorridos en campo. Estas diferencias se deben a que la densidad de plantación está en función de las condiciones ambientales, el tipo de sistema de cultivo, el manejo del tejido productivo y, en particular, del tipo de variedad cultivada. Las densidades de plantación en los cafetales estudiados varían de 2000 a 3000 cafetos por ha, siendo el promedio de 2768 plantas, lo que coincide con los resultados de otros estudios realizados en Chiapas (Escamilla et al. 2005).

\section{La transformación agroecológica en los cafetales afectados por roya}

La transformación agroecológica se basó en el manejo de diversidad de especies vegetales y el diagnóstico de la estructura productiva para determinar la renovación con otras variedades de cafetos, con el fin de mejorar la productividad.

\section{Diversidad de especies}

El menor número de especies se presentó en Comitán y el mayor en Ocozocoautla, mientras que el promedio fue de cinco especies (Cuadro 2), este valor fue menor al reportado por Escamilla et al. (2005), quienes registraron un promedio de 8.6 especies, lo que apunta a una disminución en el número de las mismas.

El promedio de cobertura de sombra fue de 56 $\%$, el mayor porcentaje se registró en Ocozocoautla, Mapastepec y Yajalón con 74, 67 y 68 \% respectivamente; además, este tipo de manejo repercute en la densidad de siembra, registrándose mayor número de árboles en las regiones con más especies, lo cual aumenta el porcentaje de sombra en los sistemas. Ruelas-Monjardín et al. (2014) señalaron que el dosel de sombra en el cafetal aumenta la complejidad y la biodiversidad de los sistemas, lo que reduce la vulnerabilidad a la roya.

Todos los productores entrevistados cultivan la especie arábica (Coffea arabica) y un grupo pequeño (2.9\%) además cultiva y produce café Robusta (Coffea canephora), que se localiza en las zonas bajas de las regiones de Copainalá, Motozintla y Tapachula. Cada productor cultiva entre dos y tres variedades de Coffea arabica, la mayoría de ellas mezcladas y de diferentes edades. En el Cuadro 3 se presentan las variedades identificadas y distribuidas por zonas altitudinales en el estado de Chiapas.

Se puede apreciar que la variedad Bourbon, a pesar de la susceptibilidad a la roya, fue la más frecuente (19\%), lo que se atribuye a su adaptabilidad a zonas con altitud >1000 msnm, así como por su buen nivel de producción y calidad (Zamarripa y Escamilla, 2016). Típica es otra variedad ampliamente utilizada debido a su resistencia a la roya (Escamilla et al., 2005; Zamarripa y Escamilla, 2016). Las variedades resistentes a roya que más se cultivan son Costa Rica (8.3 \%) y Oro Azteca (7.5 \%); según Zamarripa y Escamilla (2016), ambas variedades son preferidas para introducirlas por los productores, ya que a diferencia de las variedades tradicionales de Coffea arabica, como son Bourbon, Caturra y Típica, éstas se adaptan a las condiciones ambientales de la región y tienen bajos requerimientos de mantenimiento; por su parte, las variedades mejoradas, con resistencia a roya y mayor productividad, requieren más inversión en términos de trabajo e insumos para producir (Henderson, 2019).

El grupo de otras variedades representa el $24.4 \%$ e incluye algunas que los productores desconocen, les 


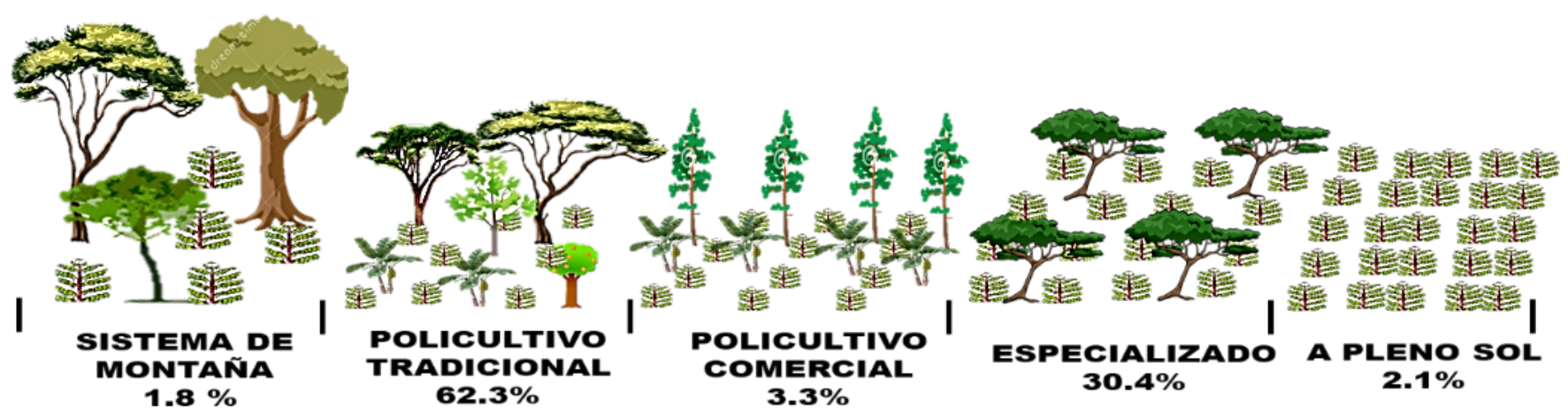

Figura 4. Sistemas de cultivo de café en Chiapas, México. Elaboración propia.

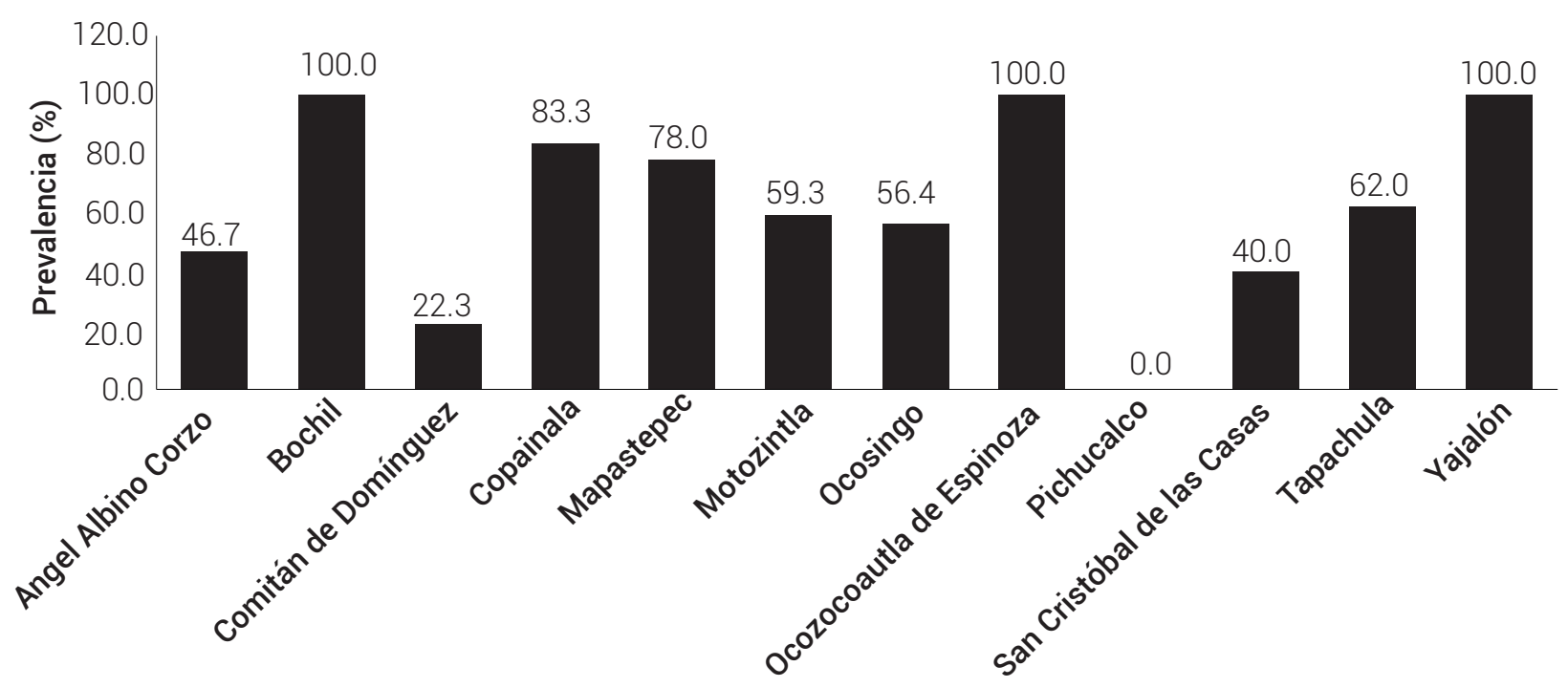

Figura 5. Porcentaje (\%) del sistema policultivo tradicional en las regiones cafetaleras de Chiapas, México.

asignan un nombre local, o bien se encuentran en bajas superficies, son los casos de Catimores no identificados, Bourbon Peñasco, Pacas, Lempira, Kaffa S12, RR y Alonso. Las regiones de Ángel Albino Corzo, Copainalá, Ocozocoautla y Tapachula poseen más del $50 \%$ de variedades tradicionales, mientras que las regiones de Comitán, Ocosingo y Yajalón presentan una mayor mezcla de variedades no identificadas o con denominaciones locales. Las variedades resistentes a la roya, como son Oro Azteca, Colombia, Costa Rica, Sarchimor, Marsellesa, Geisha y Guacamayo, en conjunto representan el 19.2\% de los cafetales estudiados. Específicamente, la variedad Sarchimor se distribuye en Mapastepec, Motozintla y Yajalón, la Marsellesa en Ángel Albino Corzo y Yajalón, la Geisha en Comitán y San Cristóbal, y la Guacamayo en
Ángel Albino Corzo. De acuerdo con Cerda et al. (2017), a partir del brote de roya en 2012, se renovaron los cafetales con variedades mejoradas resistentes, lo que ha significado una reconfiguración del patrón varietal cafetalero en el estado de Chiapas. Este cambio varietal con materiales resistentes a roya, más productivos y con mayor adaptación del cultivo a la exposición solar, ha provocado la deforestación de la sombra, modificación de las prácticas culturales, incremento de costos y mayor especialización técnica (Libert-Amico y Paz-Pellat, 2018). Esta integración de variedades mejoradas puede provocar una reducción de la calidad física del grano y café en taza, algo que ya ha sido reportado en México para estas variedades resistentes a roya (Escamilla et al., 2015). 
Cuadro 2. Número de especies arbóreas, densidad de árboles (cantidad de árboles ha-1) y porcentaje de sombra (\%)

\begin{tabular}{lccc}
\hline Región cafetalera & Especies arbóreas $^{+}$ & Cantidad de árboles $^{+\dagger}$ & Porcentaje de sombra $^{+1}$ \\
\hline Ángel Albino Corzo & 6 & 90 & 48 \\
Bochil & 4 & 64 & 52 \\
Comitán & 2 & 36 & 31 \\
Copainalá & 4 & 69 & 65 \\
Mapastepec & 5 & 80 & 67 \\
Motozintla & 6 & 97 & 51 \\
Ocosingo & 5 & 86 & 62 \\
Ocozocoautla & 8 & 124 & 74 \\
Pichucalco & 4 & 64 & 47 \\
San Cristóbal & 4 & 62 & 58 \\
Tapachula & 5 & 69 & 56 \\
Yajalón & 6 & 105 & 68 \\
Promedio & 5 & 79 & 56.6 \\
\hline
\end{tabular}

†Se determinó por unidad de estudio 1/16 de ha (625 m²). +†Se estimó en función de 1 ha. Información obtenida en los ciclos $2015-2017$.

Cuadro 3. Distribución de las variedades (\%) por estratos altitudinales en Chiapas, México.

\begin{tabular}{|c|c|c|c|c|}
\hline \multirow[b]{2}{*}{ Variedad } & \multicolumn{3}{|c|}{ Altitud (msnm) } & \multirow[b]{2}{*}{$\begin{array}{c}\text { Tota } \\
(\%)\end{array}$} \\
\hline & $\begin{array}{c}\text { Baja } \\
(<1000) \\
\end{array}$ & $\begin{array}{c}\text { Media } \\
(1000-1300) \\
\end{array}$ & $\begin{array}{c}\text { Alta } \\
(>1300) \\
\end{array}$ & \\
\hline Típica o criollo & 4.4 & 8.2 & 16.8 & 9.8 \\
\hline Bourbon & 12.1 & 25.0 & 19.8 & 19.0 \\
\hline Mundo Nuevo & 5.3 & 1.4 & 7.6 & 4.8 \\
\hline Caturra & 16.8 & 22.9 & 6.3 & 15.3 \\
\hline Garnica & 1.8 & 8.5 & 4.0 & 4.8 \\
\hline Catuai & 4.5 & 2.3 & 0.1 & 2.3 \\
\hline Pacamara & 0.0 & 0.1 & 0.2 & 0.1 \\
\hline Oro Azteca & 7.7 & 2.1 & 12.8 & 7.5 \\
\hline Colombia & 0.0 & 0.2 & 0.2 & 0.1 \\
\hline Costa Rica & 5.7 & 14.6 & 4.5 & 8.3 \\
\hline Sarchimor & 0.9 & 1.3 & 0.1 & 0.8 \\
\hline Marsellesa & 0.0 & 1.7 & 0.2 & 0.6 \\
\hline Geisha & 0.0 & 2.2 & 2.0 & 1.4 \\
\hline Guacamayo & 0.0 & 0.0 & 1.3 & 0.2 \\
\hline Otras variedades & 40.6 & 9.1 & 22.8 & 24.4 \\
\hline Robusta & 0.2 & 0.4 & 1.1 & 0.6 \\
\hline
\end{tabular}

Información generada con la base de datos de los 138 productores de los ciclos 2015-2017. 


\section{Diagnóstico de la estructura productiva de los cafetos (DEP)}

De acuerdo con la edad y las características de las plantaciones, se identificaron seis categorías (Cuadro 4).

Se aprecia que las regiones de Ocosingo y Yajalón presentan un potencial productivo alto en comparación con el resto de las regiones, pues el porcentaje de cafetos con categorías I y || suman más de $67 \%$; por su parte, Copainalá, Mapastepec, Motozintla y Tapachula son las regiones que tienen más cafetos con categorías III y IV, superando el $35 \%$. Estos resultados confirman que algunas regiones tienen niveles importantes de renovación, como Pichucalco, Yajalón Comitán y Ocozocoautla; sin embargo, en otras regiones los cafetales requieren podas y renovación.

La edad promedio de los cafetos fue de 17 años, con valores entre 2 y 70 años, lo que representa uno de los principales problemas técnicos en la mayoría de las regiones, ya que los cafetales se han envejecido a más de 20 años de edad, y el proceso de renovación ha sido lento. Los cafetales más viejos se localizan en Bochil y Mapastepec (26 años), los más jóvenes en Comitán (4 años), por lo que de acuerdo con el diagnóstico de la estructura productiva (DEP), el $29 \%$ de cafetos requieren renovación (Figura 6); aunque una tercera parte de los cafetales ya se han renovado, aún es necesario mejorar el manejo y la planeación de actividades para la mejor toma de decisiones de los productores (Díaz et al., 2013).

\section{La productividad del agroecosistema}

Durante el ciclo 2015-2016, la mayor productividad de café se registró en las regiones de Ángel Albino Corzo y Motozintla con 15.03 y $12.61 \mathrm{Qq} \mathrm{ha}^{-1}(868.73$ y $725.07 \mathrm{~kg}$ $\left.h^{-1}\right)$ de café pergamino seco respectivamente, las más bajas se registraron en Copainalá, Comitán y Ocozocoautla con 4.2, 3.52 y $2.8 \mathrm{Qq} \mathrm{ha}^{-1}\left(241.5,202.4\right.$ y $\left.161 \mathrm{~kg} \mathrm{ha}^{-1}\right) \mathrm{de}$ café pergamino seco respectivamente (Figura 7). Para el ciclo 2016-2017 se registró una recuperación a las afectaciones de la roya, que se reflejó en un incremento en la productividad de café en todas las regiones, excepto en Bochil y Motozintla. Dicho incremento se atribuye a la renovación de cafetos con variedades tolerantes a roya. Este aumento fue más importante en las regiones de Ángel Albino Corzo y Pichucalco, las cuales registraron producción promedio de 16.90 y $10.58 \mathrm{Qq} \mathrm{ha}^{-1}$ respectivamente.

\section{CONCLUSIONES}

La roya (Hemileia vastatrix) es una enfermedad que afectó la producción y transformó los agroecosistemas de café en el estado de Chiapas; en particular, integró nuevas variedades, por lo que, en algunas regiones se modificó la diversidad y estructura del componente arbóreo. La sombra y la proporción de cafetales con sistema rusticano

Cuadro 4. Categoría de cafetos por regiones cafetaleras en Chiapas, México.

\begin{tabular}{lcccccc}
\hline \multirow{2}{*}{ Región } & \multicolumn{7}{c}{ Categoría de cafetos (\%) } \\
\cline { 2 - 7 } & $\mathrm{I}$ & $\mathrm{II}$ & $\mathrm{III}$ & $\mathrm{IV}$ & $\mathrm{V}$ & $\mathrm{VI}$ \\
\hline Ángel Albino Corzo & 32.29 & 18.86 & 22.86 & 10.29 & 15.71 & 0.00 \\
Bochil & 10.86 & 47.43 & 4.57 & 28.57 & 4.00 & 4.57 \\
Comitán & 47.69 & 22.46 & 7.69 & 2.77 & 19.08 & 0.31 \\
Copainalá & 9.33 & 32.00 & 46.67 & 8.00 & 8.00 & 2.00 \\
Mapastepec & 6.40 & 40.00 & 37.60 & 0.00 & 14.40 & 1.60 \\
Motozintla & 11.63 & 37.88 & 35.50 & 3.25 & 12.13 & 0.25 \\
Ocosingo & 39.74 & 29.33 & 17.44 & 1.13 & 11.49 & 1.13 \\
Ocozocoautla & 40.44 & 21.33 & 23.11 & 7.56 & 4.89 & 2.67 \\
Pichucalco & 58.67 & 0.00 & 0.00 & 33.33 & 8.00 & 0.00 \\
San Cristóbal & 22.67 & 29.33 & 14.40 & 20.53 & 11.73 & 1.33 \\
Tapachula & 19.20 & 36.00 & 12.00 & 24.00 & 8.00 & 0.80 \\
Yajalón & 48.89 & 18.22 & 3.56 & 22.67 & 6.22 & 0.44 \\
Total & 28.98 & 27.74 & 18.78 & 13.51 & 10.30 & 1.26 \\
\hline
\end{tabular}

Categorías: I (normales), II (requieren poda), III (requieren recepa), IV (requieren renovación), V (cafetales pre-productivos), VI (cafetales con fallas físicas). Información generada con la base de datos de los 138 productores de los ciclos 2015-2017. 


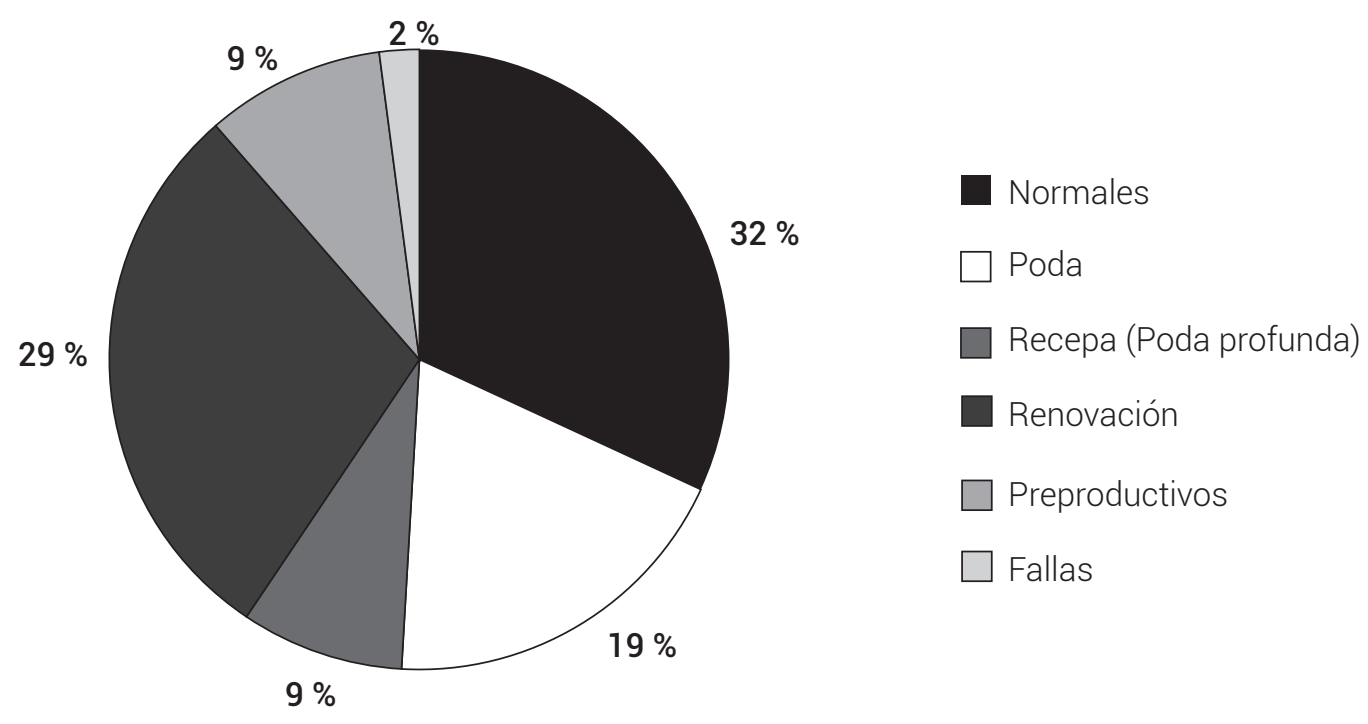

Figura 6. Categoría de cafetos (\%) en el estado de Chiapas, México.

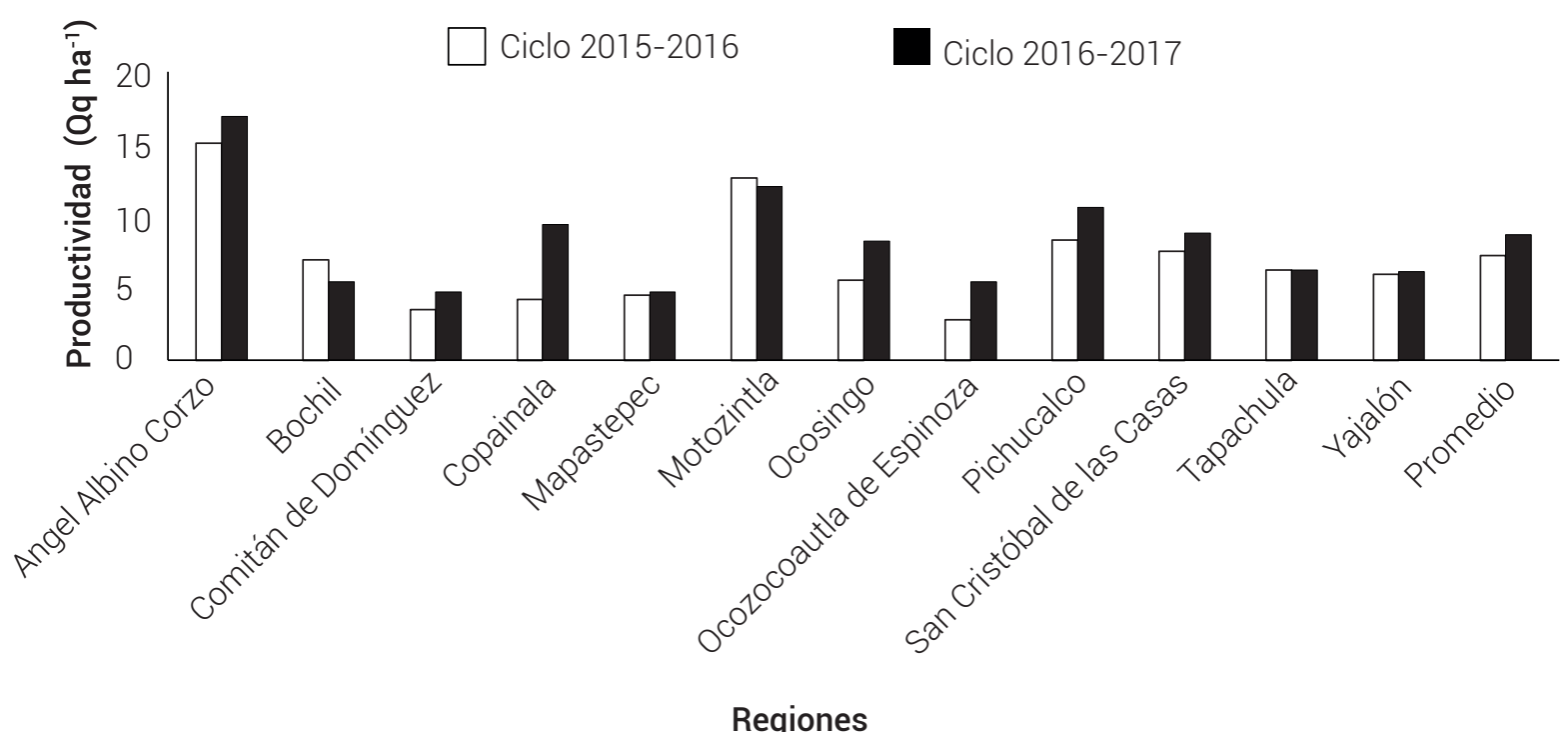

Figura 7. Promedio de productividad $\left(Q_{q}\right.$ ha $\left.^{-1}\right)$ por regiones cafetaleras en Chiapas, México. Un Qq equivale a $57.7 \mathrm{~kg}$ de café pergamino seco. 
(SR) se redujo, y se incrementaron los sistemas de policultivo tradicional y sistema especializado de manera regionalizada; sin embargo, los sistemas rusticano, sistema en policultivo comercial y sistema a pleno sol aún tienen presencia. Esta distribución de sistemas novedosos - más tradicionales, según las regiones productoras, condicionan la distribución varietal del café, por lo que hay regiones que prefieren las variedades tradicionales sobre las introducidas recientemente. La afectación de la roya al café, generó los agrupamientos de variedades: i) variedades tradicionales (Bourbon, Típica, Mundo Nuevo, Caturra, Catuai, Garnica y Pacamara), ii) Catimores (Oro Azteca, Costa Rica, Colombia y Lempira), iii) Sarchimores (Sarchimor, Marsellesa y Guacamayo) y iv) otras (Geisha, Pacas, Alonso, RR, Bourbon Peñasco, Bourbon Negro, Kaffa S12). Las agrupaciones de estas variedades responden a diversas estrategias adoptadas por los productores, la mayoría buscan la productividad y resistencia a roya, y otras están en función de la demanda de los mercados de especialidad. La recuperación de la productividad y las características agroecológicas del agroecosistema café en Chiapas evidencian que son sistemas resilientes a problemas fitosanitarios como la roya.

\section{AGRADECIMIENTOS}

Se agradece a los productores y organizaciones de Chiapas por su invaluable apoyo. Al Instituto de Café de Chiapas, a la Universidad Autónoma Chapingo y al Consejo Nacional de Ciencia y Tecnología - México.

\section{BIBLIOGRAFÍA}

Cerda R., J. Avelino, C. Gary, P. Tixier, E. Lechevallier and C. Allinne (2017) Primary and secondary yield losses caused by pests and diseases: assessment and modeling in coffee. PLOS ONE 12:e0169133, https://doi.org/10.1371/journal.pone.0169133
Díaz C. S., C. Solábac, E. Escamilla, V. Morales, E. Ameca, R. Ávila y G Castillo (2013) Estimación de cosecha de café. In: Estimación de Rendimientos en el Sector Agropecuario. Serie Estudios Críticos del Desarrollo. J. Aguilar Á. y V. H. Santoyo C. (coords.) Universidad Autónoma Chapingo y Miguel Ángel Porrúa México, D. F. pp:87-112

Escamilla P. E., O. Ruiz R., G. Díaz P., C. Landeros S., D. E. Platas R., A. Zamarripa C. y V. A. González H. (2005) El agroecosistema café orgánico en México. Manejo Integrado de Plagas y Agroecología (Costa Rica) 76:5-16.

Escamilla P. E., O. Ruiz R., A. Zamarripa C. y V. A. González H. (2015) Calidad en variedades de café orgánico en tres regiones de México. Revista de Geografía Agrícola 55:45-55,

Escamilla P. E. y S. Díaz C. (2016) Sistemas de Cultivo de Café en México. SAGARPA, COFUPRO, CENACAFÉ, Universidad Autónoma Chapingo. Xalapa, Veracruz. $63 \mathrm{p}$.

Henderson T. P. (2019) La roya y el futuro del café en Chiapas. Revista Mexicana de Sociología 81:389-416.

INCAFECH, Instituto del Café de Chiapas (2020) Datos importantes de café. Instituto de café de Chipas. Instituto de café de Chiapas. Gobierno de Chiapas. México. https://incafech.gob.mx/ cafedatos.html (Noviembre 2021).

Libert-Amico, A. y F. Paz-Pellat (2018) Del papel a la acción en la mitigación y adaptación al cambio climático: la roya del cafeto en Chiapas. Madera y Bosques 24:e2401914, https://doi. org/10.21829/myb.2018.2401914

Merlin-Uribe Y., F. Charbonnier, A. Contreras-Hernández, 0. B. Herrera H. y L. Soto-Pinto (2018) Tipología de estrategias campesinas en la caficultura orgánica de la Sierra Madre de Chiapas Ecosistemas y Recursos Agropecuarios 5:411-423, https://doi org/10.19136/era.a5n15.1714

Moguel P. and V. M. Toledo (1999) Biodiversity conservation in traditional coffee systems of Mexico. Conservation Biology 13:11-21 https://doi.org/10.1046/j.1523-1739.1999.97153.x

Naylor L. (2017) Auditing the subjects of fair trade: coffee, development and surveillance in highland Chiapas. Environment and Planning D: Society and Space 35:816-835, https://doi org/10.1177/0263775817694031

Ruelas-Monjardín L. C., M. E. Nava-Tablada, J. Cervantes y V. L. Barradas (2014) Importancia ambiental de los agroecosistemas cafetaleros bajo sombra en la zona central montañosa del estado de Veracruz, México. Madera y Bosques 20:27-40, https://doi.org/10.21829/myb.2014.203149

Vargas V. P. (2007) Mujeres cafetaleras y producción de café orgánico en Chiapas. El Cotidiano 22:74-83.

Zamarripa C. A. y E. Escamilla P. (2016) Variedades de Café en México. Origen, Características y Perspectivas. SAGARPA, COFUPRO, CENACAFÉ, Universidad Autónoma Chapingo. Xalapa, Veracruz. $43 \mathrm{p}$ 
